\title{
Controlled Multiple-Object-Dropping Mechanism Using Single Radio Channel in UAVs
}

\author{
Gaurang Gupta \\ Mechanical Engineering, Visvesvaraya National Institute of Technology, Nagpur, India
}

\begin{abstract}
The conventional way of dropping any object from a UAV (Unmanned Ariel Vehicle) can be just used once as the servo-mechanized system rotates and the object is dropped but another object cannot be loaded for the next drop when aircraft is airborne (for e.g. dummy missile under the wing). Secondly, when multiple objects are dropped from the fuselage, it's not in a controlled manner (as all drop at once). Thirdly, the number of objects to be dropped requires the equal number of radio channels for the operation, which restricts the number of objects, which can be dropped (as we have limited number of radio channels). This paper explains how to design such a mechanism to overcome these three practical issues by the virtue of which,objects, when placed in the fuselage of the aircraft can be dropped one after another in a controlled manner using a single radio channel as a control.
\end{abstract}

Keywords: Bomb, Dropping, Mechanism, Object, RC-plane, UAV

\section{Introduction}

This paper is aimed at developing new mechanisms in UAVs that can facilitate controlled and economic methods for dropping circular objectsfrom UAVs. With further work, this mechanism can be used for dropping objects of all shapes.

The conventional way of dropping any object from a UAV can be just used once. The servomechanized system rotates and the object is dropped,for example a door-opening system in the fuselage, mountedobjects under the wing, etc. These systems have a huge aerodynamic drawback, opened doors and wing mountings produce large drag wasting fuel energy. Opened door also disturbs the airflow under the aircraft affecting the natural stability of the aircraft.

The problem with drop-typewing-mount mechanism is that another object cannot be reloaded for second drop when the aircraft is airborne. Secondly if multiple objects are placed for dropping from a door-type mechanism, the drop of individual object cannot be controlled i.e. all objects will drop at oncewhen the drop is initiated. When missile-like objects are mounted under the wing, the drop is controlled but not economic. Equal number of radio channels is required as that of the number of missile-like objects to be dropped.

Hence the global objectives behind the mechanism design are to design a mechanism, which facilitates controlled dropping action of dropping one object at a time; handle multiple objects when airborne; and all these to be achieved by using a single radio channel.

\section{Design Procedure}

To solve all the above-mentioned problems, we need such a design, which will allow only one object to drop at a time by stopping all other objects when the door is open to drop one object.Shut the gate as soon as one object is dropped and back to original position to drop the next object. A four bar chain mechanism can achieve these objectives.

\subsection{Design of four bar chain}

Techniques used to synthesis a four bar chain mechanism [2] [3]: -

1. Finite Position based synthesis

2. Design using relative pole method

\subsubsection{Finite Position Designation}

We know two positions, initial and final desired position of the door. The door in its shut position is horizontal, holding the object over itself that will be dropped. The final position of the door is vertical and is inward stopping the other objects from falling out. The positions are shown in Fig. 1, red color line being the door. 


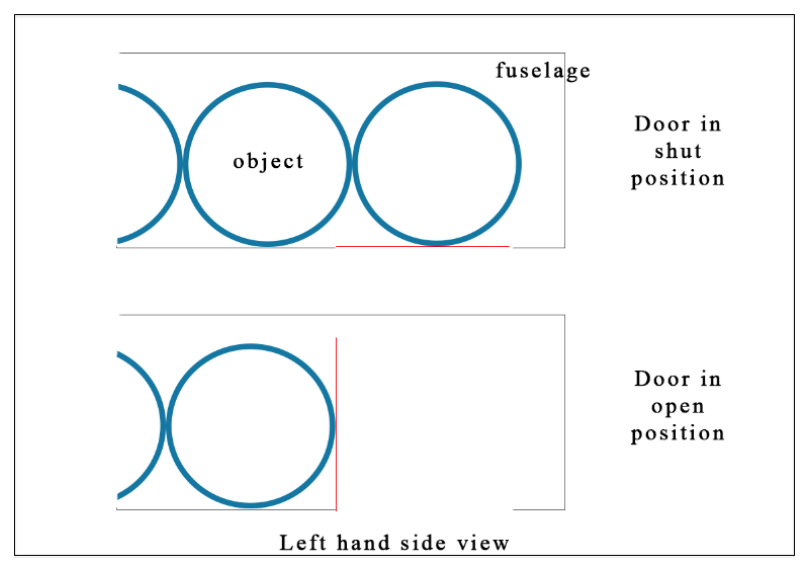

Figure 1: Shut and open door positions as desired

\subsubsection{Synthesis using Relative Pole Method}

The process began by positioning the two-desired positions of the plate (i.e. initial horizontal and final vertical position). The two end points named as $\mathrm{A} 1$ and $\mathrm{B} 1$ in the initial position and $\mathrm{A} 2$ and $\mathrm{B} 2$ in the final position as shown in Fig. 2. Then we drew perpendicular bisector to the line joining A1 and A2, similarly for B1 and B2. These two perpendicular bisectors are the loci of the relative poles of the rotating link, the servo arm and the connecting rod. The position of the relative poles will decide the position of the pivot points of the servo arm and the connecting rod.

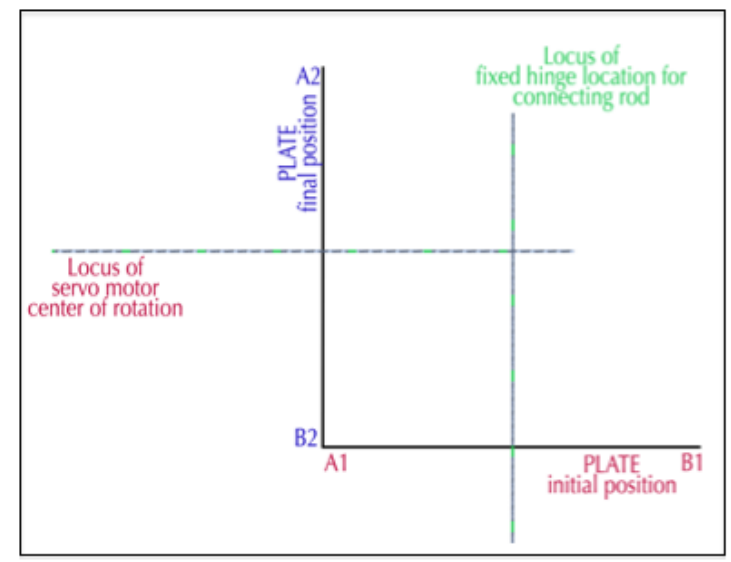

Figure 2: Relative pole Locus

After the design of the basic mechanism, displacement analysis was done on Catia and then several dimension and position changes were obtained. Once the desired position of servo arm movement within limit were achieved, as shown in Fig. 3; relation of relative lengths between links were then deduced so that it can be easily adopted else where by just amplifying few basic dimensions.

This solves our problem. The door movement being inward, the airflow affected is minimal as compared to the door opening outwards. Secondly, only one object is dropped, the one that sat on the door, when the door was shut. The final vertical position is such that it stops the objects behind from falling out, giving control on dropping of individual object individually. As a single servomotor is used, only one radio channel is used for this purpose to drop any number of objects.

\subsection{Deriving Link Dimensions (Length)}

The length of the links cannot be a decimal valve. It would create problems in manufacturing and may lead to unnecessary dimensions errors. We need to keep in mind few of the mechanical and space constraints such as all the links are to be inboard at any position during the rotation of the mechanism. Mechanical Constraints: -

1) Initial plate position.

2) Rotation angle of servo arm $90^{\circ}$

3) Final position of the plate. 


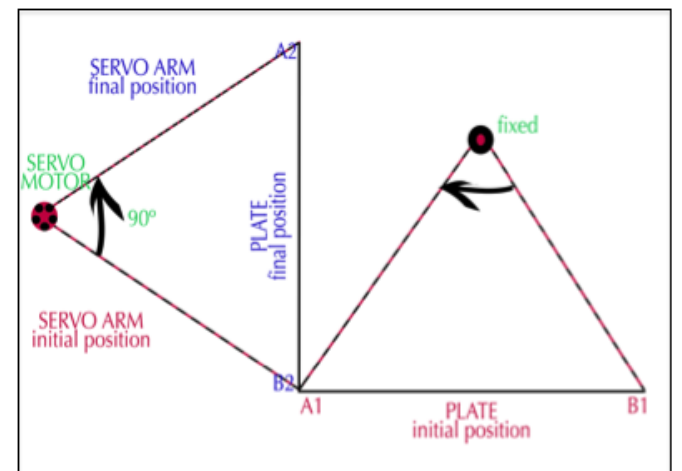

Figure 3: Mechanism in shut and open position

We have a (infinite) $)^{\wedge} 2$ system i.e. two basic parameters have to obtained; servo pivot and connecting rod pivot. A preliminary mechanism design was obtained with plate length of $5 \mathrm{~cm}$. As the servo arm was considered to rotate $90^{\circ}$, to get the pivot position, a simple property of a circle was used where, when two lines from the either ends of the diameter intersect on the circumference, they makes an angle of $90^{\circ}$ at the circumference. The dimensions got from here are the length of the servo arm required and the (x,y) position of the servo arm pivot. The pivot point for the connection rod is decided such that it does not exceed the height of the fuselage.

To keep things simple to decide, all the links were given dimensions such that the sides of their respective triangle will form a right angle triangle and hence Pythagorean-triplets is the best option.

This decision of assigning the link lengths leads to a small violation of initial assumption, that is the servo arm will rotate $90^{\circ}$, but in reality depending on the Pythagorean triplets, this angle will vary and can be later adjusted by the servo trimsetting option. To reduce the angle of rotation, the length of the servo arm can be increased and vise versa.

We hence deduce, the plate size of length $6 \mathrm{~cm}$. When the plate is in the vertical position, the half of it size is considered and hence the length of servo arm (hypotenuse) is acquired to be $5 \mathrm{~cm}$ and the servo-pivot is placed at a distance of $4 \mathrm{~cm}$ to the left of the plate, forming a Pythagorean triplet 5-4-3.

Now the connecting rod length can be considered to be $13 \mathrm{~cm}$ with its pivot approximated to be 12.5 $\mathrm{cm}$ above the midpoint of the plate. Here 13-12.489-3 approximately forms a Pythagorean triplet. We thereby come to a final mechanism as shown in Fig. 4.

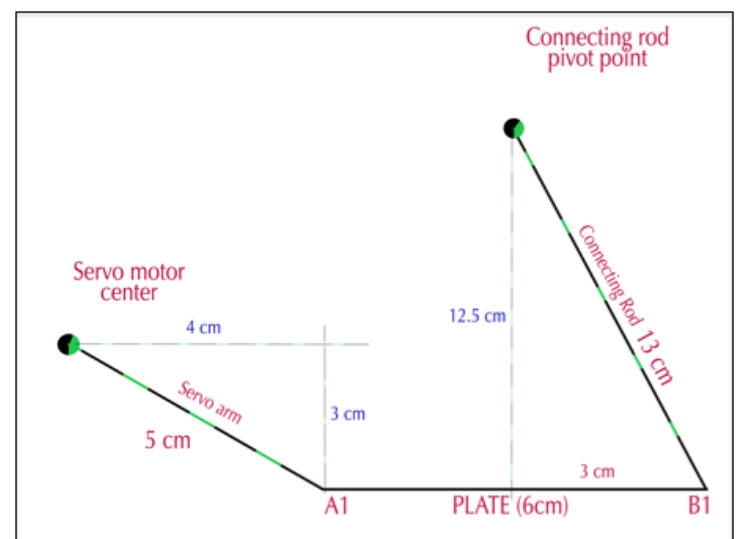

Figure 4: Final Mechanism

\section{Analysis And Simulation}

Aerodynamic flow simulations [1] were carried out which helps us determine the pressure force exerted over the plate at various positions and second. The dynamic analysis of the mechanism was then done to determining the angular acceleration, angular velocity and the angular displacement of the two hinges at the end of the plate and the trend of how the servo would exert torque.

\subsection{Aerodynamic Analysis}

Pressure force normal to the doorplate was simulated at differingAOAs and differing air velocities. AOA varied from $-15^{\circ}$ to $20^{\circ}$ with an increment of $5^{\circ}$ and velocity varied from $5 \mathrm{~m} / \mathrm{s}$ to $25 \mathrm{~m} / \mathrm{s}$ with an increment of $5 \mathrm{~m} / \mathrm{s}$. Fig. 5 shows few of the simulated results of the pressure force with varying AOA at $15 \mathrm{~m} / \mathrm{s}$. 


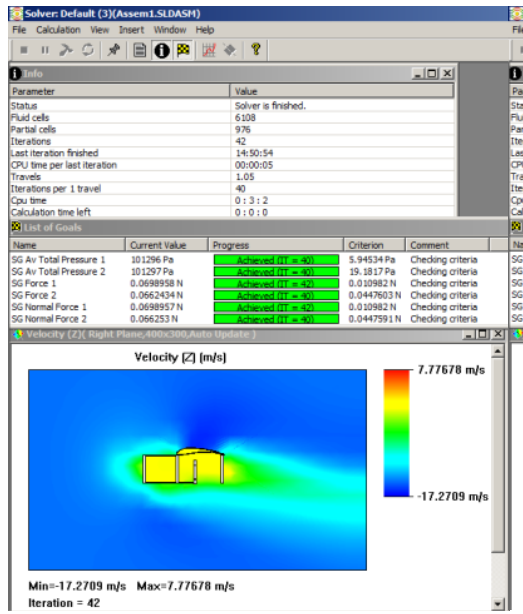

(a) $\mathrm{AOA}=-15^{\circ}$

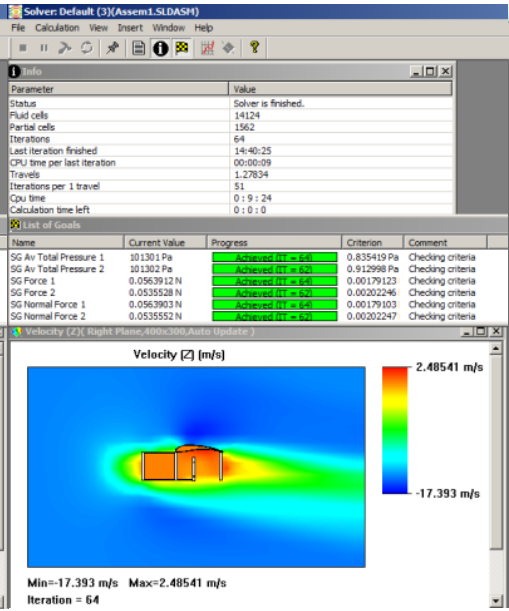

(b) $\mathrm{AOA}=-10^{\circ}$

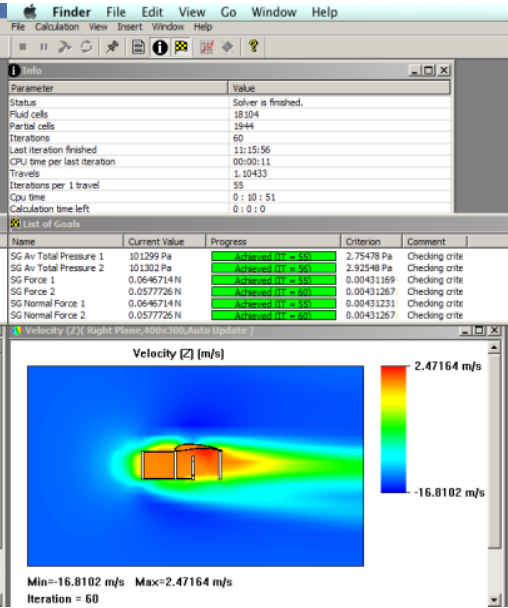

(c) $\mathrm{AOA}=-5^{\circ}$

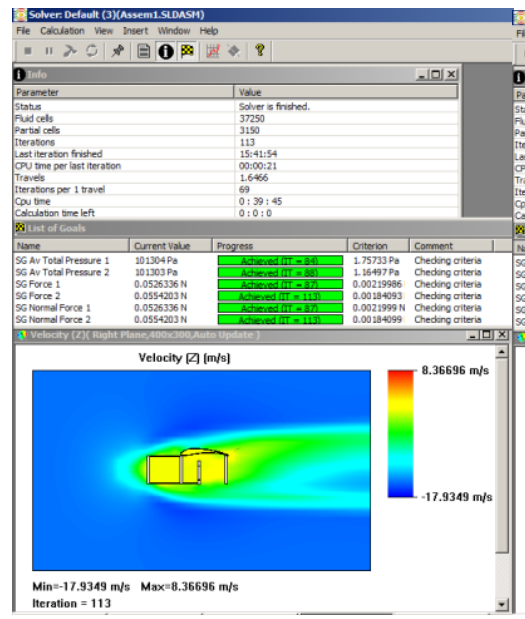

(d) $\mathrm{AOA}=\overline{0 \text { degree }}$

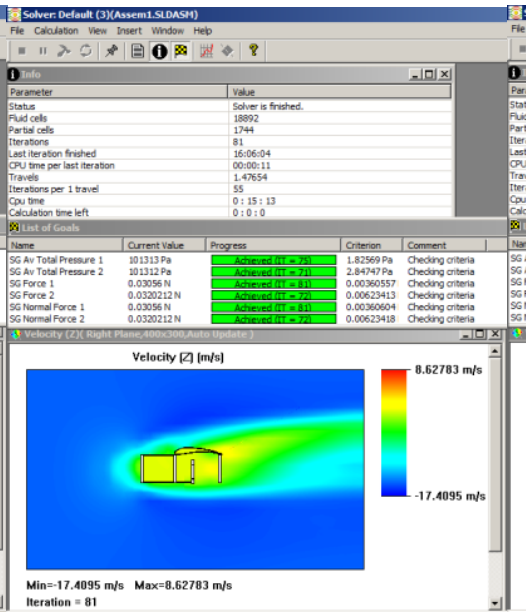

(e) $\mathrm{AOA}=5^{\circ}$

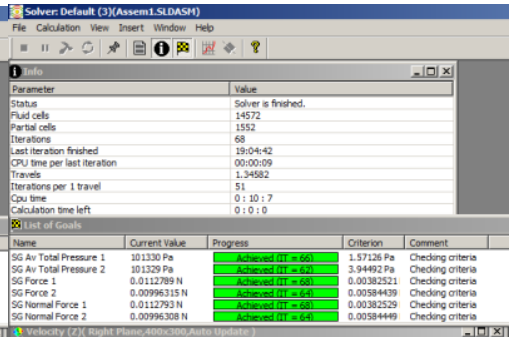

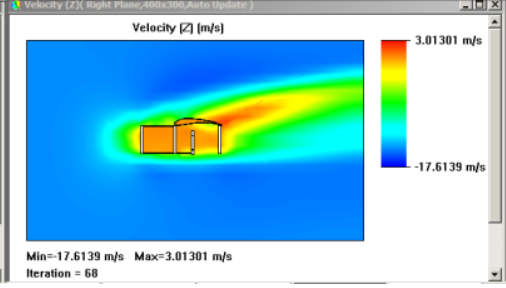

(f) $\mathrm{AOA}=10^{\circ}$

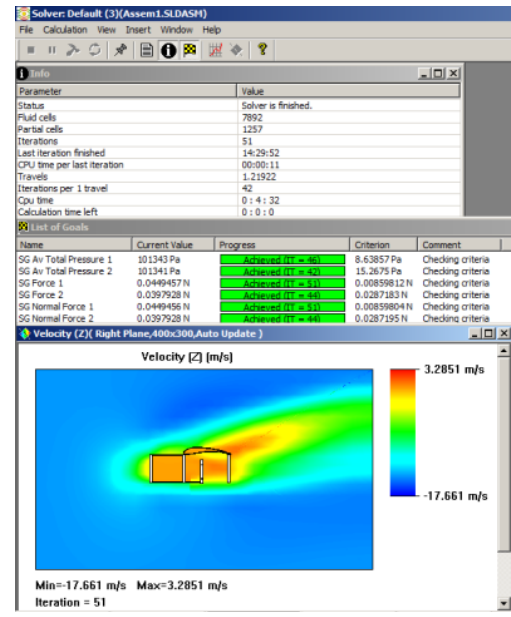

(g) $\overline{\mathrm{AOA}}=15^{\circ}$

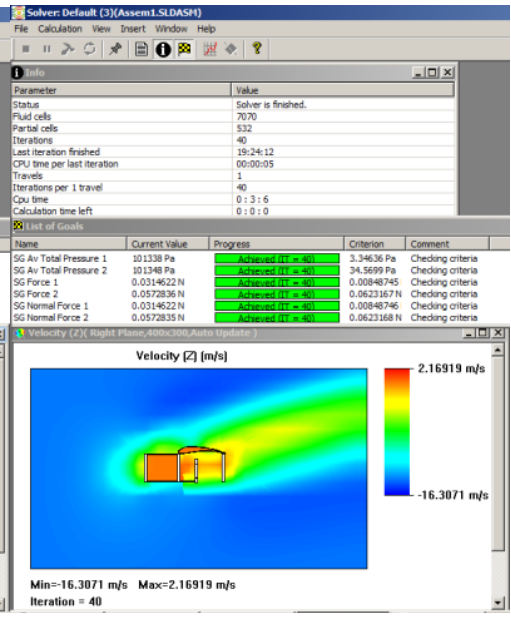

(h) $\mathrm{AOA}=20^{\circ}$

Figure 5: Aerodynamic Flow Simulations over Doorplate

Performing a total of 40 flow simulations, we get the final results, as shown in Table 1and Table 2, of the pressure force acting perpendicular to the door-plate at differing AOAs and velocity values. From these values we derive the pressure difference in which actually interests usis formulated in Table 3 . 
Table 1: Pressure force perpendicular to outside of the plate in units $10^{-6} \mathrm{~N}$

\begin{tabular}{|r|r|r|r|r|r|r|r|r|}
\hline Surface 1 & -15 & -10 & -5 & 0 & 5 & 10 & 15 & 20 \\
\hline 5 & 8714 & 8081 & 8282 & 7148 & 4368 & 279 & 3906 & 2667 \\
\hline 10 & 31029 & 29115 & 29608 & 23652 & 13769 & 4638 & 19003 & 13460 \\
\hline 15 & 69896 & 56390 & 64671 & 52634 & 30560 & 11279 & 44946 & 31462 \\
\hline 20 & 125214 & 101897 & 114537 & 95149 & 49356 & 21307 & 81444 & 56471 \\
\hline 25 & 190384 & 158306 & 176559 & 146352 & 75171 & 36171 & 128863 & 90459 \\
\hline
\end{tabular}

Table 2: Pressure force perpendicular to outside of the plate in units $10^{-6} \mathrm{~N}$

\begin{tabular}{|r|r|r|r|r|r|r|r|r|}
\hline Surface 2 & -15 & -10 & -5 & 0 & 5 & 10 & 15 & 20 \\
\hline 5 & 8317 & 7735 & 7554 & 7374 & 4524 & 76 & 3202 & 5474 \\
\hline 10 & 29397 & 27885 & 26678 & 24917 & 14387 & 3901 & 16346 & 25080 \\
\hline 15 & 66253 & 53555 & 57773 & 55420 & 32021 & 9963 & 39793 & 57284 \\
\hline 20 & 117999 & 97016 & 102111 & 101111 & 52216 & 18997 & 71316 & 105271 \\
\hline 25 & 180034 & 151350 & 157935 & 154852 & 79258 & 31712 & 113953 & 162882 \\
\hline
\end{tabular}

Table 3: Net Force (by pressure difference) perpendicular to the plate in units $10^{-6} \mathrm{~N}$

\begin{tabular}{|r|r|r|r|r|r|r|r|r|}
\hline DIFFERENCE & -15 & -10 & -5 & 0 & 5 & 10 & 15 & 20 \\
\hline 5 & 397 & 346 & 728 & -226 & -156 & 203 & 704 & -2807 \\
\hline 10 & 1632 & 1230 & 2930 & -1265 & -618 & 737 & 2657 & -11620 \\
\hline 15 & 3643 & 2835 & 6898 & -2786 & -1461 & 1316 & 1915 & -25822 \\
\hline 20 & 7215 & 4881 & 12426 & -5962 & -2860 & 2310 & 10128 & -48800 \\
\hline 25 & 10350 & 6956 & 18624 & -8500 & -4087 & 4459 & 14910 & -72423 \\
\hline
\end{tabular}

Figure 6 shows the graphical representation of air pressure forces acting perpendicular to doorplate surfaces. Figure 7 shows the difference in the pressure force acting on the plate i.e. the net force acting on the plate.

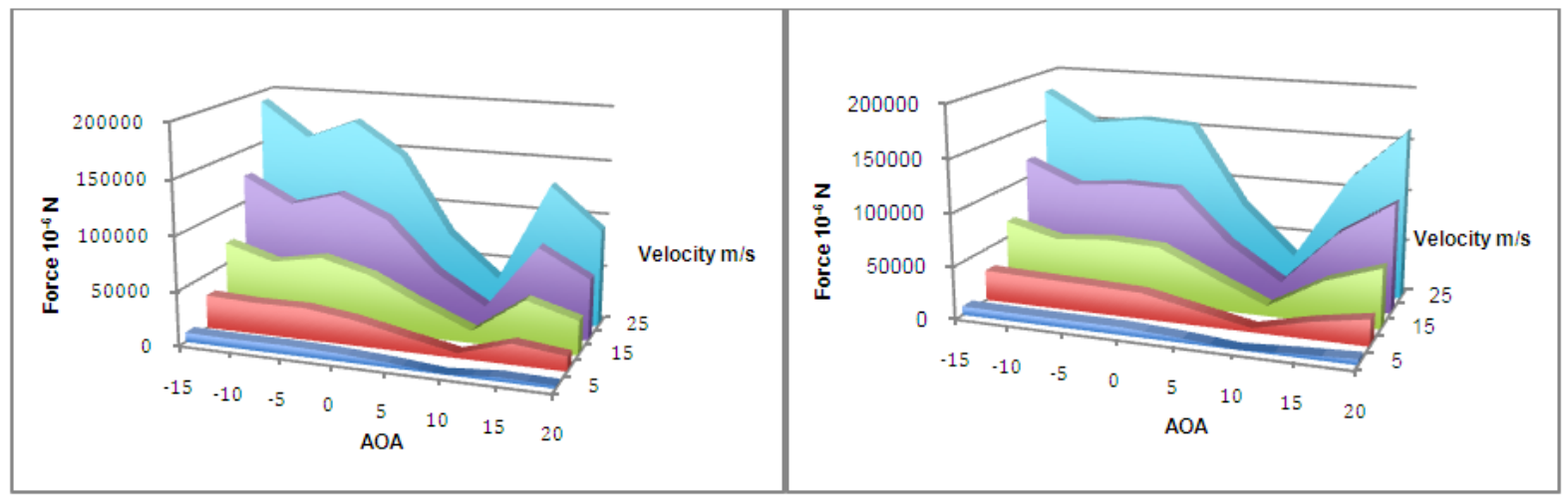

(a) Surface 1

(b) Surface 2

Figure 6: Forces Acting Perpendicular to Plate Surfaces

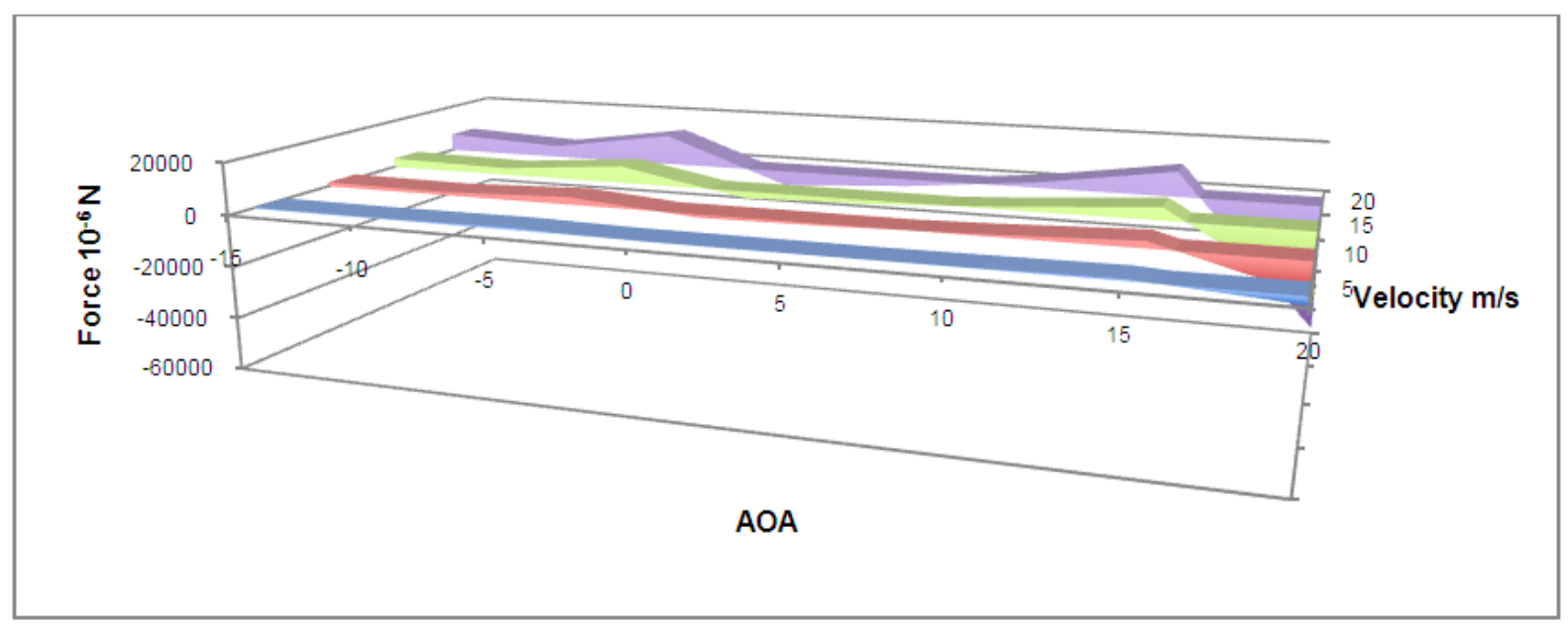

Figure 7: Net Force Acting on the Plate 


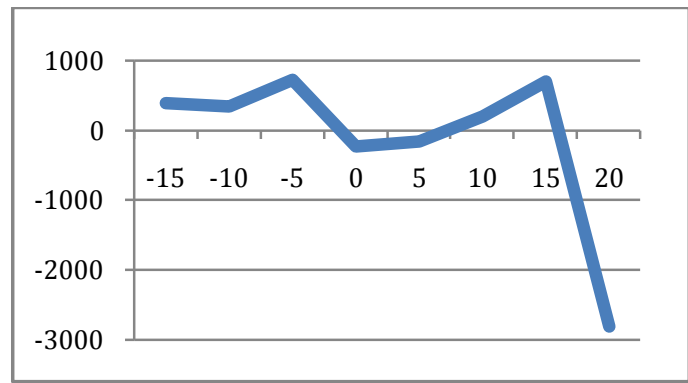

(a) velocity $5 \mathrm{~m} / \mathrm{s}$

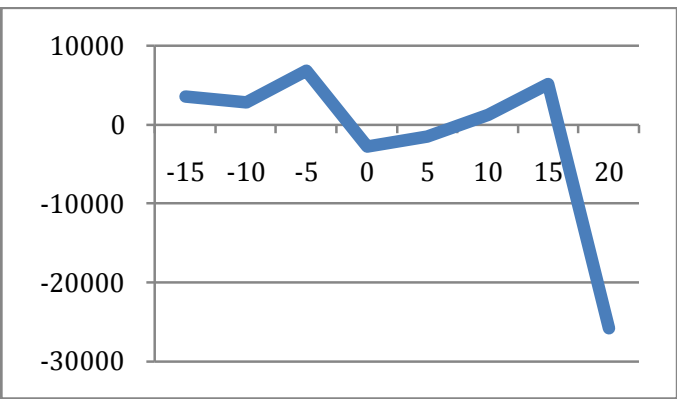

(c) velocity $15 \mathrm{~m} / \mathrm{s}$

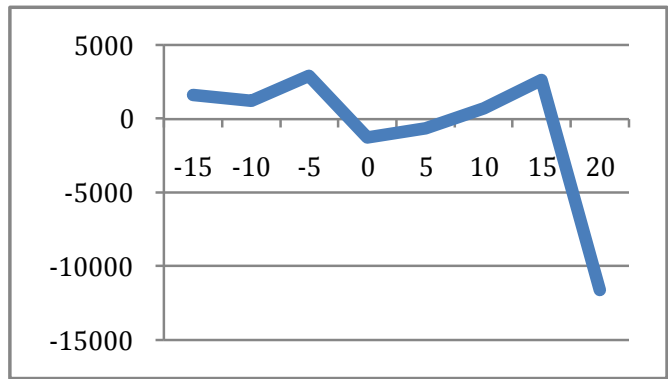

(b) velocity $10 \mathrm{~m} / \mathrm{s}$

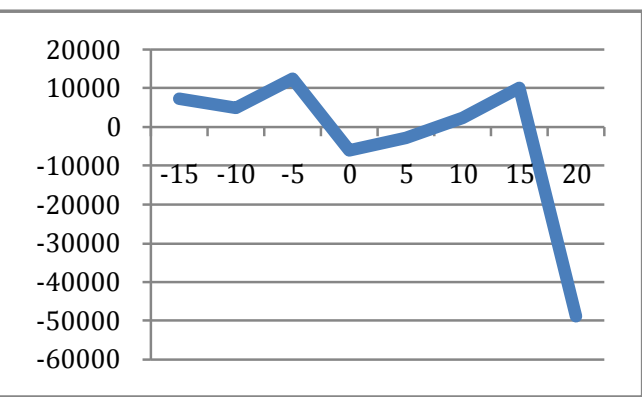

(d) velocity $20 \mathrm{~m} / \mathrm{s}$

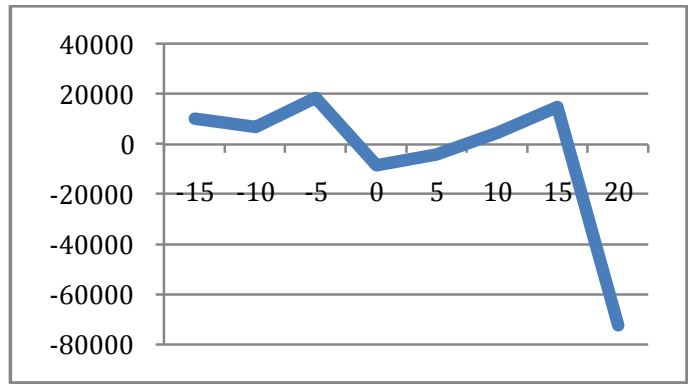

(e) velocity $25 \mathrm{~m} / \mathrm{s}$

Figure 8: Force $\left(10^{-6} \mathrm{~N}\right)$ varying with $\mathrm{AOA}$ at given velocities

Figure 8 shows how the trend in the graph is followed as force varies with AOA at given velocities. We get two significant observationsfrom all the values obtained from the simulations. 1) There is a sudden drop in pressure force values between $\mathrm{AOA}$ of $5^{\circ}$ and $10^{\circ} ; 2$ ) The trend of the graph of the net force exerted on the plate is independent of velocity.

From the first point it can be inferred that the suddenfall in the value of force occurs because the airfoil used, s1223, reaches its maximum Lift to Drag ratio close to an $\mathrm{AOA}=7^{\circ}$ producing least drag and hence less air disturbance behind the wing and hence less pressure change effect over the plate. We further use another airfoil, this time symmetric, to validate the above stated point. The airfoil used is NACA 0020 having its maximum Lift to Drag ratio close to $\mathrm{AOA}=6^{\circ}$. Results shown in Fig. 9 .

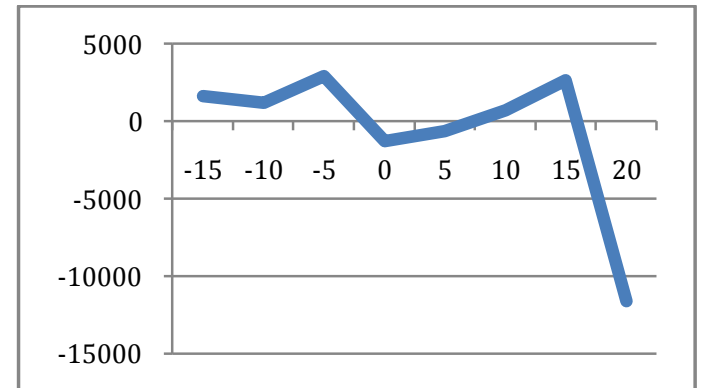

(a) velocity $=10 \mathrm{~m} / \mathrm{s}$

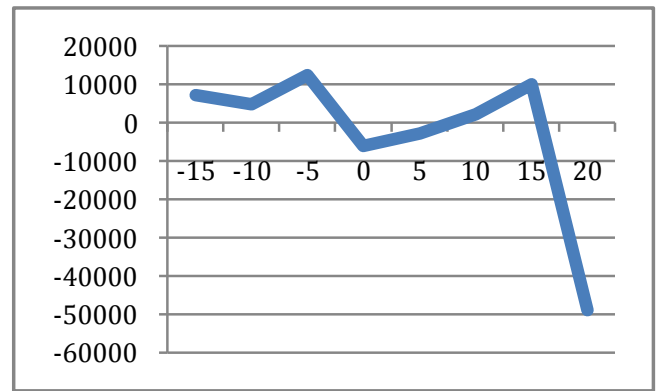

(b) velocity $=20 \mathrm{~m} / \mathrm{s}$

Figure 9: Net force $\left(10^{-6} \mathrm{~N}\right)$ variation on the door plate with varying AOA for NACA 0020 


\subsection{Dynamic Analysis of mechanism}

Dynamic analysis of the mechanism is performed to generate the basic trend of variation in magnitudes of angular displacement, angular velocity and angular acceleration verse time of the two trans-rotational hinges A1 and B1 (Fig. 4) [4] [5]. General trend of variation of torque required verses time of servo motor is also found out, pivoted at servo motor center point (Fig. 4).Time lapse to open the door is roughly calculated to be is 0.6 seconds.

Below stated are the observations and inferences we deduce from the general dynamics of hinge points (as shown in Fig. 10) and the torque required by the servomotor (as show in Fig. 11).

1) At hinge A1, variation of angular displacement verses time is smooth almost through out but as soon as the as the door is about to reach its final position, there is a sudden change in the slope of the plot, as its only the magnitude plot. The angular displacement beyond this 'Zero' point becomes positive as it continues to rotate (Fig. 10, Plot 1).

2) At hinge A1, variation of angular velocity verses time is smooth through out the operation as hinge A1 basically traces a circle.

3) At hinge A1, in variation of angular acceleration verses time plot, there occurs a huge peak. The peak occurs when the object hits the opposite wall of the aircraft fuselage and sudden reaction comes on the hinge.Anothersmall peak is observed when the object is dropped off the plate and there is sudden loose of force on the plate which is seen on the hinge.

4) At hinge B1, there exists point where magnitude of both angular velocity and angular displacement become zero. This is a point after which, there is a change in the direction of rotation of this hinge from counter clock wise to clock wise (Fig. 10, Plot 2).

5) In the motor torque diagram (Fig. 11); we observe there occur two huge peaks. The first peak occurs when the object hits the opposite wall of the aircraft fuselage and the motor encounters sudden increase in load. Another peak is observed when the object is dropped off the plate and there is sudden lose of force on the plate and motor encounters sudden decrease in load.

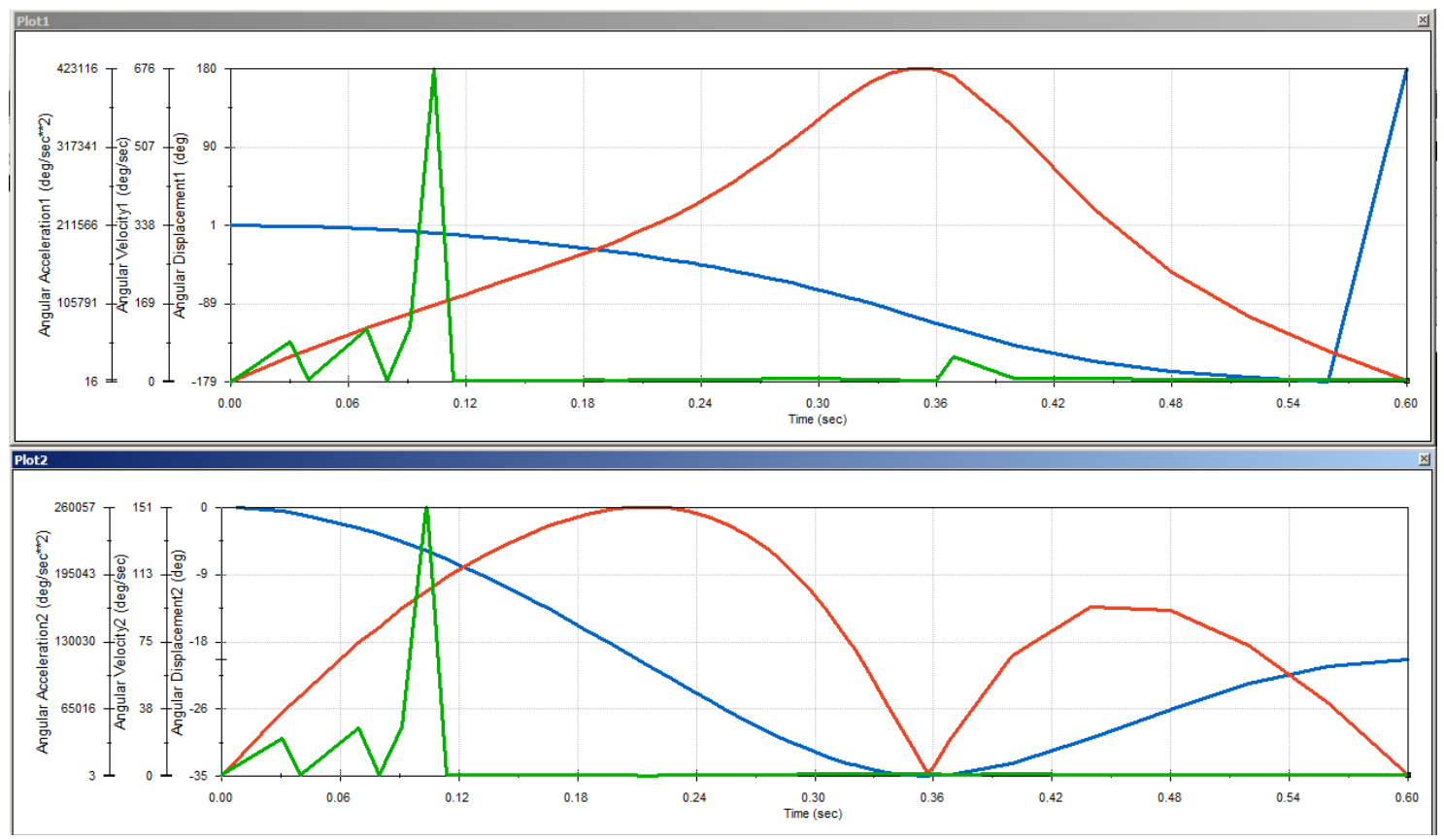

Figure 10: Dynamics of hinge points (Plot 1: hinge A1; Plot 2: hinge B1)

Key: - Blue -angular displacement (magnitude)

Red -angular velocity (magnitude)

Green -angular acceleration (magnitude) 


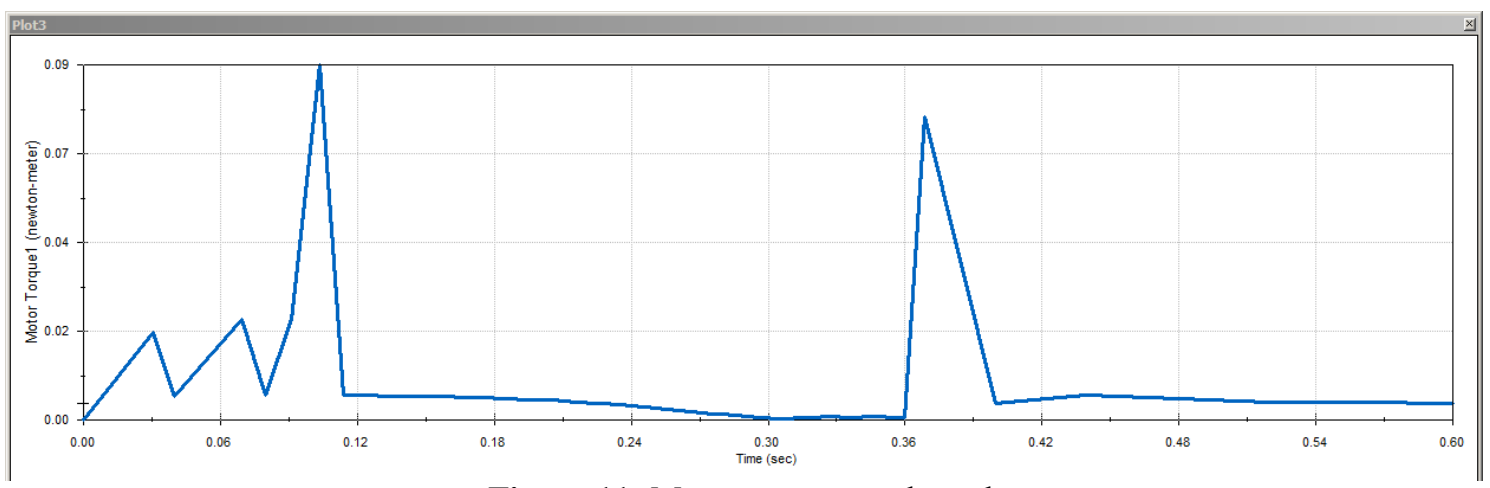

Figure 11: Motor torque trend graph

\section{Conclusion}

The advantages of this paper, as stated above, are that by using a single a single radio channel, we can control the release of multiple objects. Secondly, the design and fabrication of the dropping mechanism is very simple as it is just a four bar chain mechanism. The major limitation of this system is that it is suitable for round shapes object. Few modifications will have to be made to make it suitable for other shapes. Only the solution to limited radio channels is covered from the issue mentioned of objects thatare placed under the wing. The solution for reloading under the wing is not covered in this paper.Principle of inertia is taken into consideration by the virtue of which reloading is done in this system designed. This system was designed keeping in the mind its application in a UAV where its helps drop an object and simultaneously restricts rest of the objects from falling out.It can be used in places where a human has to control the process flow of material in desired quantity where number of actuators is limited.

\section{Acknowledgement}

The idea for this paper was inspired from the flower dropping system, which already exists. It would not have been possible to present this entire paper without the help of Dr. Shital Chiddarwar, ma'am, who instructed and helped me throughout the process. Flight instructors, Mr. Zubin and Mr. Niraj helped me with the aerodynamics required. Special thanks to Ms. Nehal Saraf and Mr. Utkarsh Garg for their help and support.

\section{Books:}

\section{Reference}

[1]. John Matsson, An Introduction to SolidWorks Flow Simulation 2014 (SDC Publications; July 10, 2014)

[2]. J. Michael McCarthy, Gim Song Soh, Geometric Design of Linkages (Springer; 25 November 2010)

[3]. Asok Kumar Mallik, Amitabha Ghosh, Gunter Dittrich, Kinematic Analysis and Synthesis of Mechanisms (Taylor \& Francis Group; 1995)

[4]. An Introduction to Motion Analysis Applications with SolidWorks Motion, Instructor Guide, (SolidWorks Education, Engineering Design and Technology Series)

[5]. Kuang-Hua Chang Ph. D., Motion Simulation and Mechanism Design with SolidWorks Motion 2013, (SDC Publications) 